\title{
A Survey on Depiction of Image using Tone and Sketch
}

\author{
Shubhavardhini P.N, Srinidhi M.S, Swathi P. S, Vindya Bhat, Rajath A. N* \\ Department of CSE, GSSSIETW, Mysuru, Karnataka, India
}

DOI: https://doi.org/10.21467/proceedings.1.42

* Corresponding author email: rajathan@gsss.edu.in

\begin{abstract}
This paper proposes a pencil drawing generating system that can create various pencil drawing styles for images, to satisfy different needs of users. The results contain various natural strokes and patterns and are structurally representative. They are accomplished by novelty combining the tone and stroke structures, which complement each other in generating visually constrained results. Prior knowledge on pencil drawing is also incorporated, making the two basic functions robust against noise, strong texture, and significant illumination variation. In light of edge, shadow, and shading information conveyance, our pencil drawing system establishes a style that artists use to understand visual data and draw them. Meanwhile, it lets the results contain rich and well-ordered lines to vividly express the original scene.

Keywords-Pencil drawing, non-photo realistic rendering, tonal drawing, gradient
\end{abstract}

\section{INTRODUCTION}

The pencil drawing depicts that human perception of abstract things is closely related to the visual perception of nature and is one of the most basic expressions in the art of painting. At present, the drawing technology of pencil drawing is divided into three-dimensional space and image space. The purpose of the three-dimensional space is to treat it as a stylized scene or model. Among them, the representative pencil drawing method based on the $3 \mathrm{D}$ model is realized by simulating the texture synthesis, drawing method and drawing step [2, Lee etal., 2006]. With the popularity of digital cameras and internet sharing, obtaining high-quality pictures is much easier than constructing 3D models of a scene. Therefore, the researchers are more inclined to study pencil drawing simulation technology based on the two-dimensional model. While there has been working to produce line drawings [DeCarlo et al. 2003; Judd et al.2007; Lee et al.2007; Grabli et al.2010], hatching [Hertzmann and Zorin 2000; Praun et al. 2001], and a combination of them [Lee et al. 2006], it is still difficult for many methods to produce comparable results to those with $3 \mathrm{D}$ model guidance. It is because accurately extracting and manipulating structures, which is almost effortless using 3D models with known boundary and geometry, becomes challenging due to the existence of texture, noise, and illumination variation.

Firstly, to capture the essential characteristics of pencil sketch and simulate rapid nib movement in drawing, we put stroke generation into a convolution framework, which differs

\footnotetext{
(C) 2018 Copyright held by the author(s). Published by AIJR Publisher in Proceedings of the $3^{\text {rd }}$ National Conference on Image Processing, Computing, Communication, Networking and Data Analytics (NCICCNDA 2018), April 28, 2018.

This is an open access article under Creative Commons Attribution-NonCommercial 4.0 International (CC BY-NC 4.0) license, which permits any non-commercial use, distribution, adaptation, and reproduction in any medium, as long as the original work is properly cited. ISBN: 978-81-936820-0-5
} 
A Survey on Depiction of Image using Tone and Sketch

our method from existing approaches [Judd et al. 2007; Grabli et al. 2010]. Secondly, to avoid artifacts caused by hatching, we bring in tonal patterns consisting of dense pencil strokes without dominant directions. Parametric histogram models are proposed to adjust the tone, leveraging the statistics from a set of sketch examples. Finally, an exponential model with global optimization is advocated to perform tone adjustment, notably benefiting rendering in heavily textured regions and object contours. The resulting pencil drawing combines sketchy strokes and tonal drawings, which well characterize and approximate the common two-step human drawing process [Wang 2002]. All these steps in our framework play fundamental roles in constructing a simple and yet very effective drawing system with a single natural image as input.

\section{OVERVIEW OF DEPICTION OF IMAGE}

Our image-based pencil sketching approach consists of two main steps, i.e., pencil stroke generation and pencil tone drawing. Their effects complement each other. Specifically, stroke drawing aims at expressing general structures of the scene, while the tone drawing focuses more on shapes, shadow, and shading than on the use of lines. The framework is illustrated in Fig. 1.

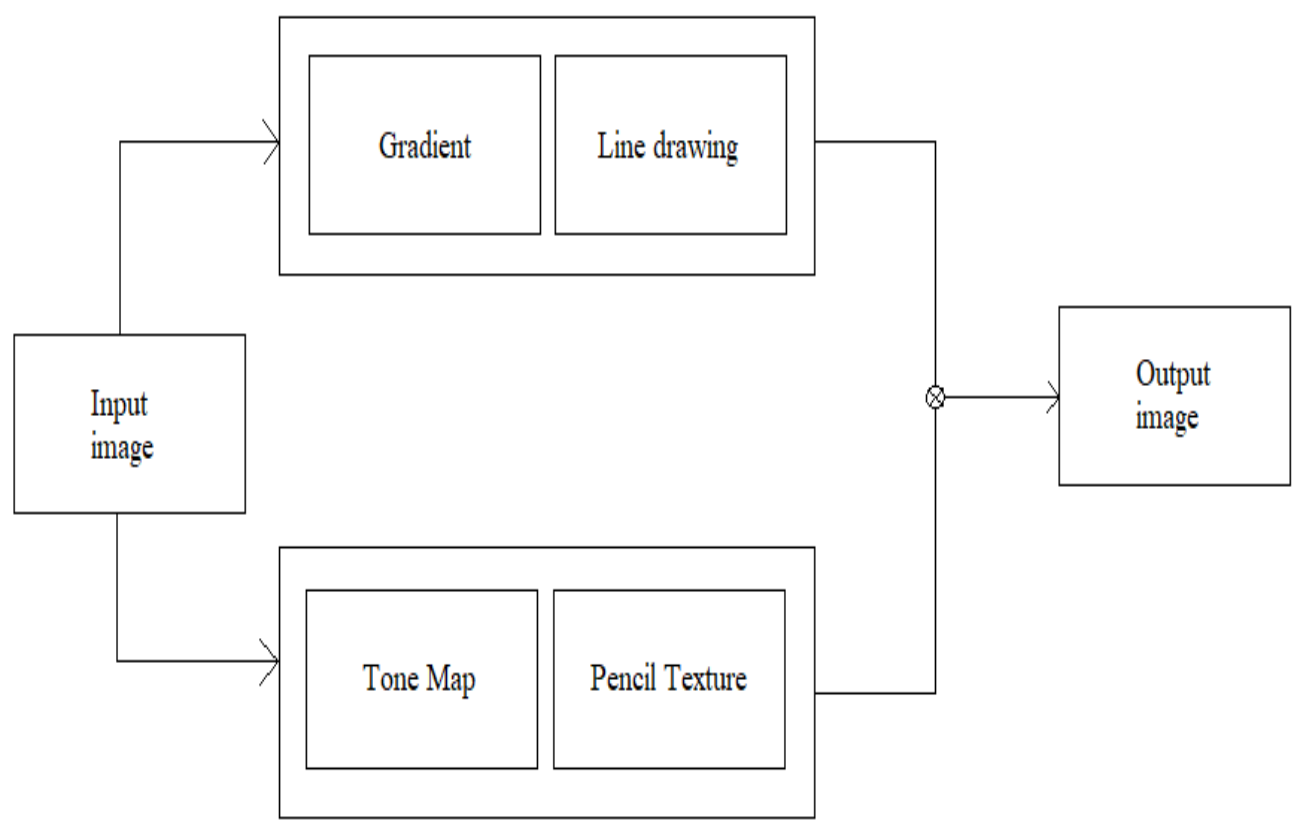

Fig.-1: Overview of Image Depiction

\subsection{Line Drawing with Strokes}

Strokes are the vital elements in line drawing. It is easy to find that artists cannot always draw very long curves without any break. Strokes end often at points of curvature and junctions. Consequently, there might be crosses at the junction of two lines in human drawing, caused 
by rapid hand movement. First, the input color image is transformed into gray scale image, and then the gradient map is obtained. The gradient map is filtered in eight directions, and the maximum response of the obtained direction is convoluted again. The maximum value of the eight filtering directions is filtered again to obtain a smoothing effect. Finally, the results of the eight directions are combined to obtain the strokes.

\subsection{Tone Drawing}

Artist usually uses dense lines in order to emphasize the shadows and dark objects in the picture. Therefore, we not only need realistic lines, but also need the similar tone and texture of the pencil drawing to get a better pencil drawing. The texture rendering is started after the tone map is generated, that is, the process by which the artist repeatedly draws lines with pencils. In detail, the same texture as the artist's hand-drawn pencil drawing is obtained by simple splicing small pieces of texture. Then, we use an optimization to solve the problem.

\section{RELATED WORK}

Chunhui Zhao, Bing Gao, Weiwei Deng, and Hongyu Zhang proposed a system for A Pencil Drawing Algorithm Based on Wavelet Transform Multiscale [1]. Non-photo realistic rendering (NPR) plays an essential role in the field of graphics, which has been pursuing the simulation and reproduction of artistic styles. In recent years, more and more researchers have been attracted to process the natural images into pencil drawings. Many pencil drawing algorithms have been developed. But the existing pencil drawing algorithms including the problems of missing picture details and coursing edge lines cannot get the desired results. Therefore, a novel pencil drawing algorithm generated by multi-scale system is proposed, which is based on the artist's hand-drawn pencil drawing process.

Hwei Jen Lin and Yue-Sheng Li proposed a system for Generation of pencil sketch drawing [2]. This paper proposes a pencil sketch generating system that can create various pencil sketching styles for images, to satisfy different needs of users. The pencil sketch generating process includes process of three parts: line drawing, tone adjustment, and texture rendering. Some operations needed are first proposed, and various pencil sketching styles that generated by the proposed system are illustrated.

Ning WANG, Bao-Gang HU proposed a system for IdiotPencil: An Interactive System for Generating Pencil Drawings From 3D Polygonal Models [3]. This paper describes an interactive system, called "IdiotPencil", that enables users to design pencil drawings directly from 3D polygonal models. The potential users of the system are professional designers or non-professional users. The system will automatically generate feature strokes and provides fast and easy-to-use tools for hatching stroke design. Users only need to specify hatching carriers according to their experiences or intentions. The hatching tools can perceive hatching regions and arrange hatching strokes automatically. In this way, the system allocates most of manual, yet tedious works to the computer while retaining aesthetic control for users.

Proceedings of the $3^{\text {rd }}$ National Conference on Image Processing, Computing, Communication, Networking and Data Analytics (NCICCNDA 2018) 
A Survey on Depiction of Image using Tone and Sketch

Compared with the existing manual pencil drawing method, the system can speed up the user's drawing and reduce their drawing skill requirements. Several examples of drawings are given by the Idiot Pencil system. The compelling features are obtained from viewpoints of both handcrafted effect and high efficiency when using the computer-aided drawing system.

Pinaki Pratim Acharjya, Ritaban Das, Dibyendu Ghoshal proposed a system for A Study on Image Edge Detection Using the Gradients [4]. A study on image edge detection using gradients is presented in this paper. In image processing and image analysis edge detection is one of the most common operations. Edges form the outline of an object and also it is the boundary between an object and the background. Detecting accurate edges are very important for analyzing the basic properties associated with an image such as area, perimeter, and shape. Max Mignotte proposed a system for Unsupervised statistical sketching for non-photorealistic rendering models [5]. This paper investigates the use'of the Bayesian inference for devising on unsupervised sketch rendering procedure. As likelihood model of this inference, we exploit the recent statistical model of the gradient vector field distribution proposed by Destrempes el al. for contour detection. A global prior deformation model for each pencil stroke is also considered. In this Bayesian framework, the placement of each stroke is viewed as the search of the Maximum A Posteriori estimation of theposterior distribution of its deformations. We use a stochastic optimization algorithm in order to find these optimal deformations. This yields an unsupervised method to create realistic hand-sketched pencil drawings. Combined with an example-based local rendering model, used to transfer the textural tone value of a given depiction style, the proposed scheme allows to simulate automatic synthetic of various artistic illustration styles.

\section{CONCLUSION}

Pencil drawing from natural images is an inherently difficult problem because not only structural information should be selectively preserved but as well the appearance should approximate that of real drawing using pencils. Thus, a general algorithm that works for many types of natural images and can produce visually compelling results is hard to develop. The main contribution includes a novel sketchy line generation scheme and a tonal pencil texture rendering step. They are effective and robust.

\section{REFERENCES}

[1]. chunhui zhao, bing gao, weiwei deng, and hongyu zhang. a pencil drawing algorithm based on wavelet transform multiscale.

[2]. hwei jen lin and tue-sheng li. generation of pencil sketch drawing.

[3]. ning wang, bao-gang hu. idiotpencil:an interactive system for generating pencil drawings from $3 \mathrm{~d}$ polygonal models.

[4]. pinakai pratim acharjya, ritaban das, dibyendu ghoshal. a study on image edge detection using the gradients.

[5]. max mignotte. unsupervised statistical sketching for non-photorealistic rendering models.

[6]. bousseau,a., kaplan, m., thollot,j., andsillion,f.x. 2006. interactive watercolor rendering with temporal coherence and abstraction. 